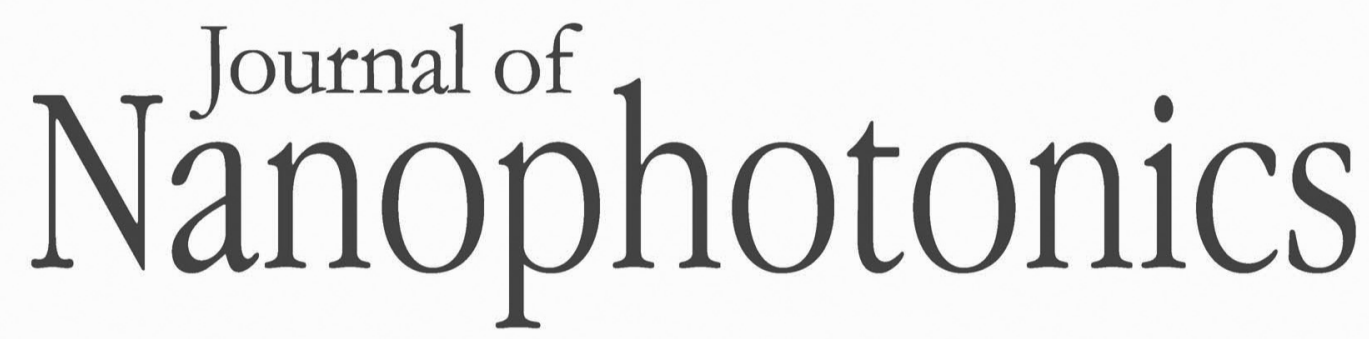

\title{
Resonance-based integrated plasmonic nanosensor for lab-on-chip applications
}

Salah E. El-Zohary

Abdulilah Azzazi

Hiroyuki Okamoto

Toshihiro Okamoto

Masanobu Haraguchi

Mohamed A. Swillam

\section{O SPIE}




\title{
Resonance-based integrated plasmonic nanosensor for lab-on-chip applications
}

\author{
Salah E. El-Zohary, ${ }^{\text {a,d }}$ Abdulilah Azzazi, ${ }^{\text {b }}$ Hiroyuki Okamoto, ${ }^{\text {c }}$ \\ Toshihiro Okamoto, ${ }^{a}$ Masanobu Haraguchi, ${ }^{a}$ and Mohamed A. Swillam ${ }^{b}$ \\ ${ }^{a}$ University of Tokushima, Department of Optical Science and Technology, \\ 2-1 Minamijosanjima-Cho, Tokushima, Tokushima 770-8506, Japan \\ elzohary@opt.tokushima-u.ac.jp \\ ${ }^{\mathrm{b}}$ American University in Cairo, School of Sciences and Engineering, Department of Physics, \\ New Cairo 11835, Egypt \\ 'Anan National College of Technology, Department of Systems and Control Engineering, \\ Anan, Tokushima 774-0017, Japan \\ ${ }^{\mathrm{d}}$ Tanta University, Faculty of Science, Physics Department, Tanta 31527, Egypt
}

\begin{abstract}
An integrated plasmonic resonator was proposed and analyzed. The detection performance of our device has been numerically verified by finite-difference time-domain simulations. The spectral sensitivity obtained was found to be $700 \mathrm{~nm} / \mathrm{RIU}$, where RIU is the refractive index unit. Our proposed sensor was found to have a detection limit in the order of $10^{-6}$ RIU. The plasmonic sensor could be fabricated using focus ion beam milling. Our design leads to an ultra-compact sensor suitable for on-chip sensing applications associated with a high sensitivity. For biosensing, the proposed sensor could have the ability for a specific capture of biomolecules at the sensor surface that enables for quantification of the biomolecules. (C) The Authors. Published by SPIE under a Creative Commons Attribution 3.0 Unported License. Distribution or reproduction of this work in whole or in part requires full attribution of the original publication, including its DOI. [DOI: 10.1117/1.JNP.7.073077]
\end{abstract}

Keywords: plasmonic resonator; surface plasmons; optical sensing; optical sensors; photodetectors; refractive index; plasmonic nanosensor.

Paper 13055 received Aug. 28, 2013; revised manuscript received Oct. 22, 2013; accepted for publication Oct. 22, 2013; published online Nov. 20, 2013; corrected May 23, 2014.

\section{Introduction}

Refractive index sensing has a wide range of applications in areas such as chemistry, physics, and biomedical engineering. Measuring the refractive indices of chemicals and food products is utilized for managing the quality of these products in the field of industry. The density and concentration of mixtures can be measured by detecting a refractive index change of the product such as chemicals. ${ }^{1-4}$

One of the most widely used approaches involves detecting the spectral shift of a resonant feature as the refractive index is varied. Different techniques have been introduced for the implementation of compact and portable sensors. ${ }^{5}$

Recently, the detection of minor changes in the refractive index has been required for precise sensing. Therefore, the refractive index measurement system with a high accuracy is of prime importance.

In the last decade, novel sensing techniques with high accuracy and good detectivity (detection limit) have been proposed based on plasmonic structures. ${ }^{6,7}$ Among these techniques, surface plasmon resonance (SPR) is considered as a key candidate for such application sensors. SPR uses surface plasmon polariton (SPP) waves to probe interactions between biomolecules, and sensor surfaces have attracted tremendous interest in the past decade for optical detection of small biological or chemical entities in liquids. ${ }^{5-7}$

In general, surface plasmon wave is formed through coherent oscillation of free electrons at a metal-dielectric interface. The electromagnetic energy of a surface plasmon mode is highly confined at the metal-dielectric interface. Thus, these modes are highly sensitive to the refractive 
index changes of the dielectric medium. ${ }^{8}$ However, conventional SPR sensor systems are usually large and bulky because of the excitation and interrogation mechanism, which is mostly done through prism coupling and angle interrogation. Thus, those systems and their associated peripherals are not well suited for lab-on-chip applications. Recently, plasmonic resonators have been investigated to propose novel integrated structures with small footprints. These plasmonic resonators can easily be influenced by the surrounding environment, which may result in shift of the resonance wavelength. ${ }^{9-13}$

In this article, we propose a novel system using the plasmonic resonator for detecting a microchange of the refractive index. The detection performance of our device has been numerically evaluated by finite-difference time-domain (FDTD) simulations. Our design can be easily fabricated using the focus ion beam milling technique. It leads to a highly compact sensor in terms of high integration in dimensions of a few hundred nanometers associated with a high sensitivity and high detection limit.

\section{FDTD Simulations}

In order to verify the performance of the proposed plasmonic sensor, commercial FDTD simulation has been exploited. ${ }^{14}$ In order to test our setup, a few test cases have been first examined by verifying the simulation results in Ref. 11. In addition, the perfectly matched layer (PML) has been optimized in order to minimize the reflection from plasmonic waveguides. Figure 1 shows a schematic of the plasmonic square resonator using metal-insulator-metal configuration. The refractive index of the $\mathrm{SiO}_{2}$ substrate is set to 1.45 . The permittivity of silver is given by the Drude model as

$$
\varepsilon(\omega)=\varepsilon_{\infty}-\frac{\omega_{\mathrm{p}}^{2}}{\omega^{2}+i \gamma \omega},
$$

where $\varepsilon_{\infty}$ is the relative permittivity at infinite frequency, $\omega_{\mathrm{p}}$ is the plasma frequency, and $\gamma$ is the collision frequency; these parameters are set to $6.0,1.5 \times 10^{16} \mathrm{rad} / \mathrm{s}$, and $7.73 \times 10^{13} \mathrm{rad} / \mathrm{s}$, respectively. ${ }^{15}$

The two-dimensional transverse magnetic simulations have been performed with a mesh size in $x$ and $y$ direction taken to be $3 \mathrm{~nm}$, number of time steps 32,000 with time step of $0.007236 \mathrm{fs}$. The PML is utilized with 12 layers in all directions. A convergence analysis has been performed to examine the effect of mesh size on the output. This analysis shows that a change of $1 \%$ is obtained for mesh size of $4 \mathrm{~nm}$.

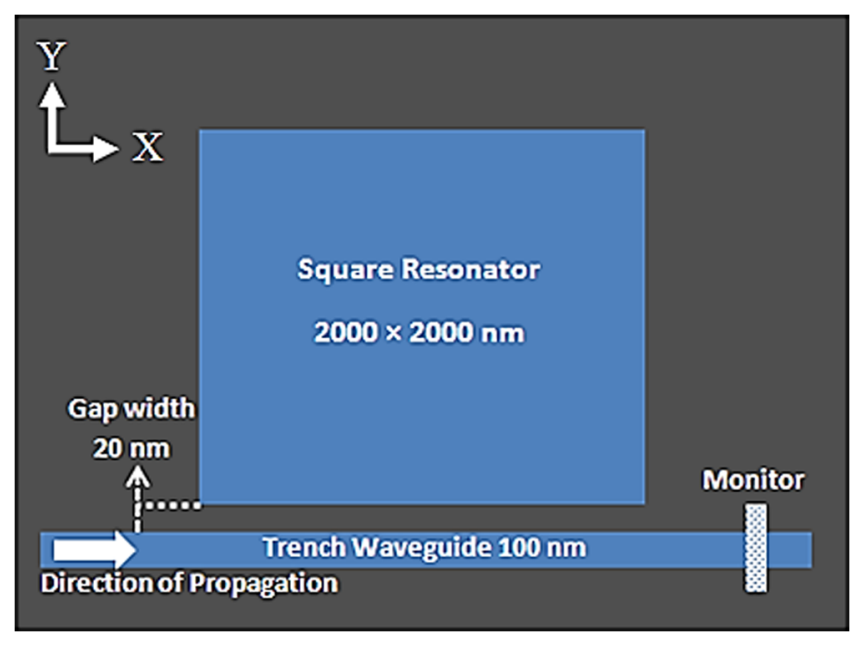

Fig. 1 Schematics of the plasmonic square resonator with a trench structure used in the finitedifference time-domain simulations. 


\subsection{Wavelength Characteristics of the Plasmonic Square Resonator}

The wavelength characteristics of the plasmonic square resonator are evaluated using FDTD. Figure 2 shows the transmission intensities $T$ (output power divided by the input power, $\left.\left|E_{\text {output }}\right|^{2} /\left|E_{\text {input }}\right|^{2}\right)$ of the trench plasmonic waveguide as a function of wavelength. The solid and dotted lines correspond to the transmission intensities when the refractive index near the resonator changes from 1.00 to 1.01 , respectively. The light is incident at the input port (denoted by direction of propagation in Fig. 1). The transmission intensity is detected around $450 \mathrm{~nm}$ away from the square resonator's right edge (denoted by monitor in Fig. 1).

For specific wavelengths, resonance occurs and plasmons are coupled to the square resonator. The transmission spectrum will exhibit a very sharp drop for this specific wavelength. The resonance wavelength is very sensitive to changes of the effective index, which is very sensitive to changes of the refractive index of the surrounding medium. ${ }^{16}$

When the refractive index of dielectric surroundings of square cavity is changed, the resonance condition for the device changes, and as a result, the resonant mode shifts to a new wavelength. Thus, a change of the refractive index of sensed materials can be detected by the shift of the resonant wavelength. In addition, change in the refractive index of the region probed by the resonant mode causes a corresponding frequency shift. The change in the resonant frequency is converted to the sensing signal. ${ }^{17}$

For our proposed sensor, it was found that when the refractive index near the resonator varies from 1.00 to 1.01 , the wavelength is shifted by nearly $7 \mathrm{~nm}$. The wavelength shift could be shown clearly in the magnified image in Fig. 2.

For high detection of refractive index changes, it is preferred to use the wavelength with the largest rate of change in the transmission intensity as an experimental reference, so that a small shift in the wavelength corresponds to the largest change in intensity. It was found that at a wavelength equal to $691 \mathrm{~nm}$, when the refractive index was changed from 1.00 to 1.01 , the transmission intensity had the maximum absolute slope. Such a drop in the transmission intensity holds the key to detect the small refractive index change near the plasmonic resonator. A shift in the resonant wavelength provides information about the refractive index shift. Thus, it is standard to define the spectral sensitivity of such sensors as $S=\Delta \lambda / \Delta n$. The spectral sensitivity has a unit of nm/RIU, where RIU is the refractive index unit. ${ }^{18}$ In our case, the spectral sensitivity was found to be $700 \mathrm{~nm} / \mathrm{RIU}$.

The impact of our proposed sensor as compact sensor suitable for on-chip sensing applications could be understood when involved in a proposed design of an integrated hybrid chip, as shown in Fig. 3. It should be mentioned that other devices can be fabricated and integrated on the same hybrid chip to serve as a multifunctional lab-on-chip. The proposed integrated plasmonic sensor will be associated with input and output ports through an efficient coupling between the plasmonic-slot waveguide and a silicon waveguide. The efficient coupling between plasmonic waveguide and silicon waveguide, recently, has been demonstrated over wide band. ${ }^{19,20}$

As shown in Fig. 3, the refractive index of dialectical surroundings of square cavity could be changed as a result of gas or liquid flow near the square resonator. Thus, our proposed sensor could be used in gas- and liquid-phase chemical sensing. Also, it is well known that the specific
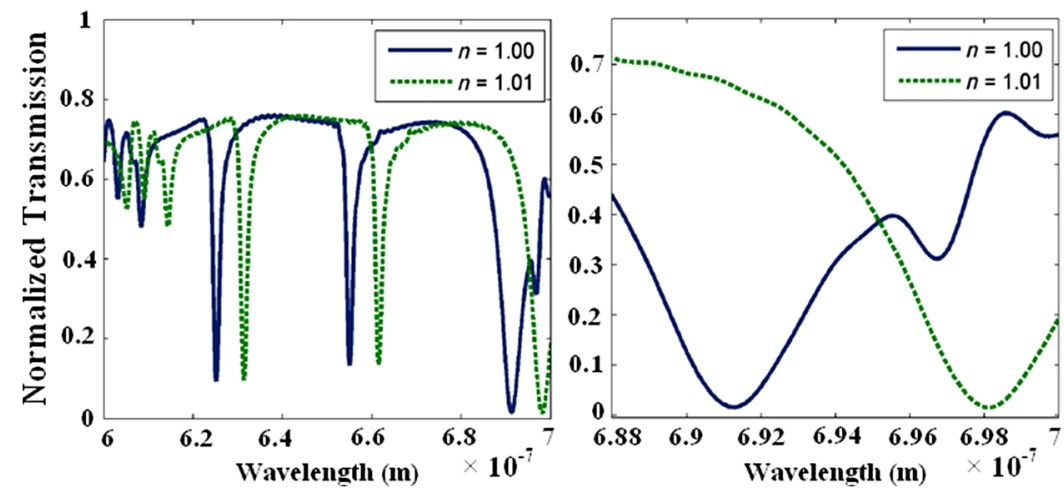

Fig. 2 Transmission spectra of plasmonic square resonator as a function of wavelength. 


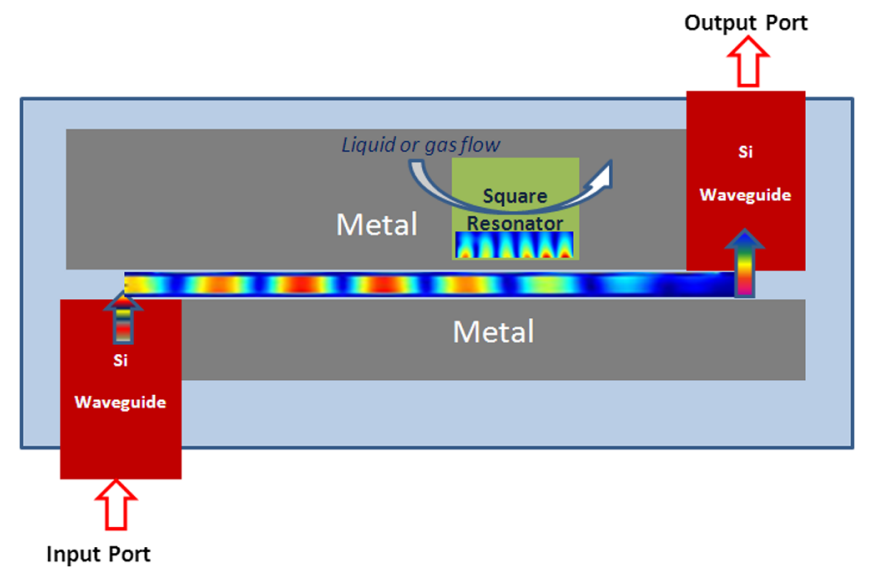

Fig. 3 A proposed design of an integrated hybrid chip involving our proposed plasmonic sensor.

capture of biomolecules at the sensor surface results in a local change in refractive index, producing a sensing signal that enables quantification of the biomolecules in a sample. ${ }^{17}$

In addition, the sensing mode of operation could use a broadband input. As a function of the refractive index of the sample medium, we monitor the position of the spectral minima in the transmission curve. This approach has been called "wavelength interrogation mode" in the literature. ${ }^{16}$

\subsection{Electric Field Profiles of the Plasmonic Square Resonator}

Figures 4(a) and 4(b) show the profiles of the electric field in the plasmonic square resonator at a wavelength of 691.0 and $673.0 \mathrm{~nm}$, respectively. As shown in Fig. 4(a) at the resonance wavelength of $691.0 \mathrm{~nm}$, SPPs are enhanced in the square resonator. The SPPs are coupled from the waveguide to the square resonator and enhanced. In this case, the transmission intensity is minimum at the output port.

In Fig. 4(b), a negligible portion of the optical field is coupled from the waveguide to the square resonator. The SPPs are not coupled in the square resonator as the wavelength of $673.0 \mathrm{~nm}$ is off-resonance wavelength.

Therefore, due to the high-field localization inside the square resonator at the resonance case, the interaction between the optical field and any material inside this resonator is maximized. Accordingly, any small change of the refractive index inside the resonator results in a significant shift in the resonance condition.
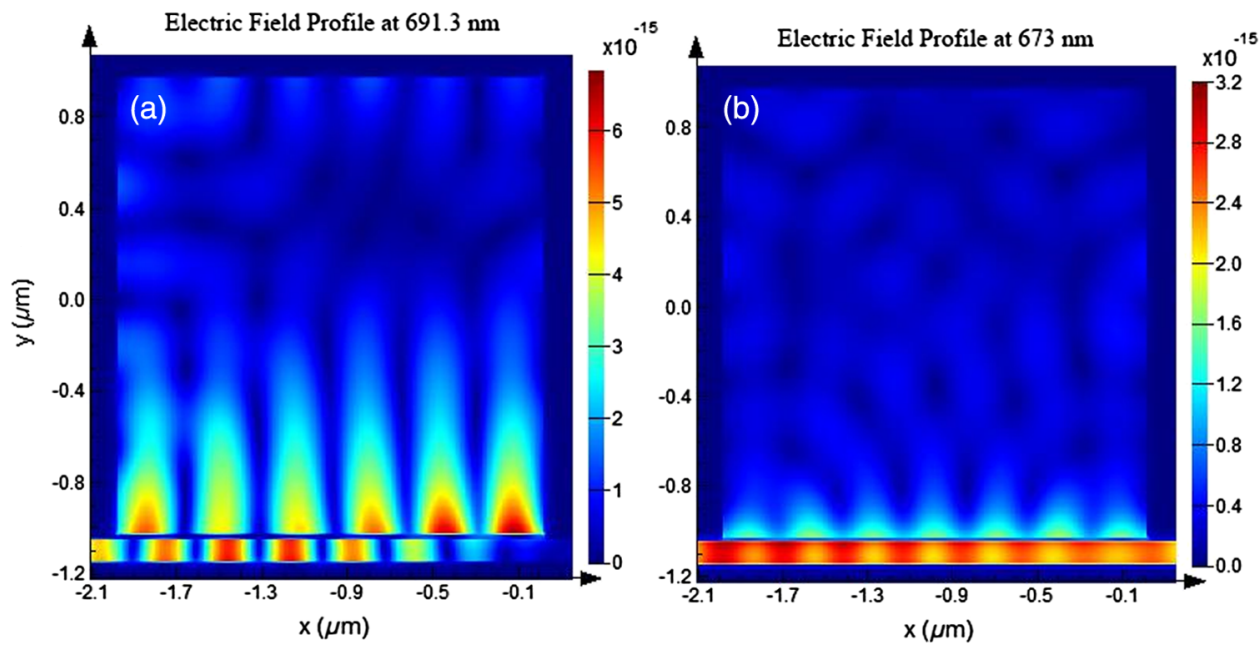

Fig. 4 Profiles of the electric field in the plasmonic square resonator for a wavelength of (a) $691.3 \mathrm{~nm}$ and (b) $673.0 \mathrm{~nm}$. 


\subsection{Analysis of the Detection Limit of the Refractive Index Change}

In order to evaluate the detection limit of the refractive index change, the refractive index of materials surrounding the plasmonic square resonator is varied from 1.0 to 1.01.

Figure 5 shows the transmission intensity as a function of the refractive index of materials surrounding the plasmonic square resonator. Figure 5 also shows a magnified view of refractive index range from 1.0 to $1.0+10^{-7}$. As shown in Fig. 5, the transmission intensity decreases when the refractive index of materials surrounding the plasmonic square resonator increases. It was found that the difference of the transmission intensity could be seen when the refractive index changed from 1.0 to $1.0+10^{-6}$.

However, the difference of the transmission intensity could not been distinguished when the refractive index changed from 1.0 to $1.0+10^{-7}$. Therefore, the detection limit of the refractive index change is $10^{-6}$. The highest detection limit of existing system is $10^{-6}$ RIU. The detection limit of our proposed plasmonic square resonator is almost same as the existing system that has the highest detection limit.

\subsection{Sensitivity Analysis of the Proposed Sensor}

In order to analyze the sensitivity of the proposed sensor to the design and fabrication parameters, various simulations have been performed. For example, the effect of the gap changes examined are shown in Fig. 6 for different gap sizes. The gap size, of course, is one of the important design parameters as it controls the amount of the power coupled to the resonator. Increasing the gap size effect causes a small shift in the resonance wavelength. It also causes deterioration in the power coupled to the resonator due to the increase of the gap size over the skin depth of the optical field inside the optical metals at the visible band, which is limited to a few tens of nanometers. Thus, it is obvious from the figure that for gap size of $>40 \mathrm{~nm}$, the resonance power starts to diminish. Thus, based on these results, a value of $<40 \mathrm{~nm}$ for the gap size is required to ensure acceptable performance of the resonator. The change in the refractive index has a negligible effect on the resonance of the proposed structure.

The effect of fabrication imperfections in the design parameters has also been investigated by examining $10 \%$ change in the gap size, as shown in Fig. 7. It is clear that a small shift in the resonance wavelength occurs due to the change in the gap size. Figure 8 also shows the response due to a change in the resonator dimension change by $10 \%$ in each direction to result in a resonator of $1.8 \times 1.8 \mu \mathrm{m}$. Based on the results from Figs. 7 and 8, it is clear that working at the wavelength of $525 \mathrm{~nm}$ is less sensitive to the fabrication of the design parameters. Thus, if the fabrication process requires high tolerance, it may be more suitable to utilize the resonance at $625 \mathrm{~nm}$. This resonance has the same sensitivity as the one at $692 \mathrm{~nm}$, as shown in Fig. 1. It also has less sensitivity to the fabrication parameters, and hence, it may be more suitable for a fabrication process with large tolerance.
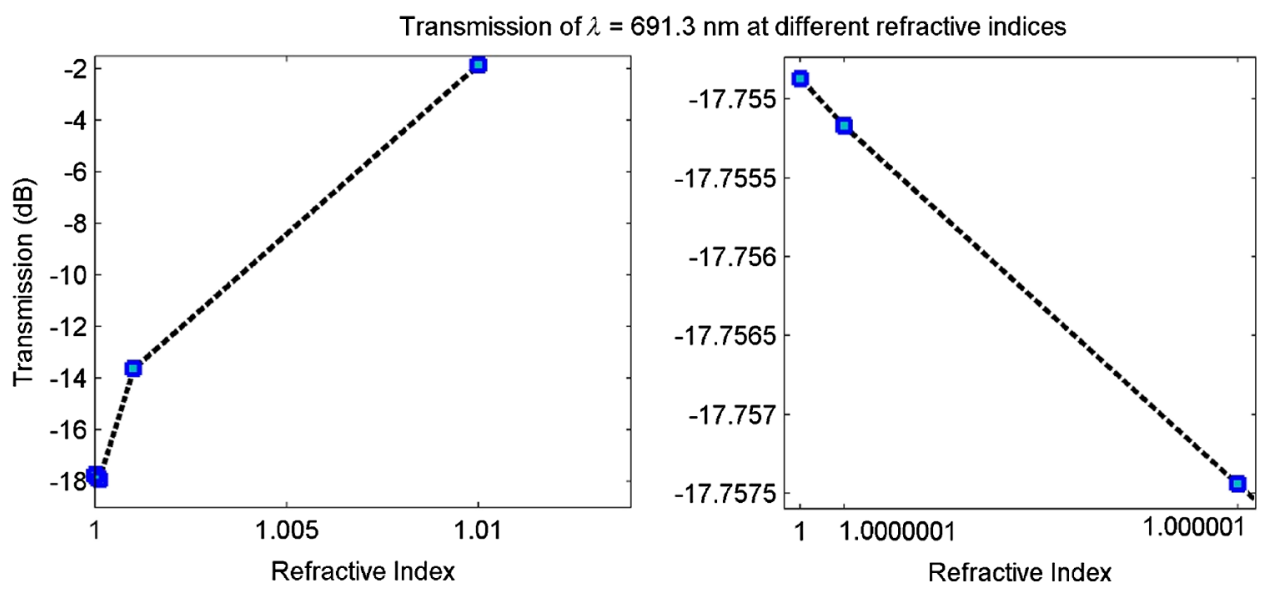

Fig. 5 Transmission intensity at the wavelength of $691 \mathrm{~nm}$ as a function of the refractive index changes of materials surrounding the plasmonic square resonator. 


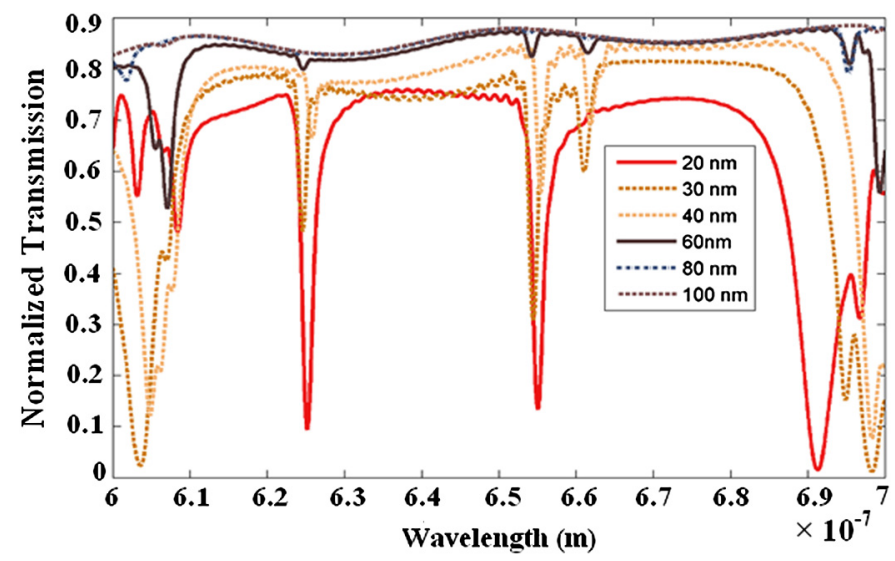

Fig. 6 Transmission intensity as a function of the wavelength for different gap sizes.

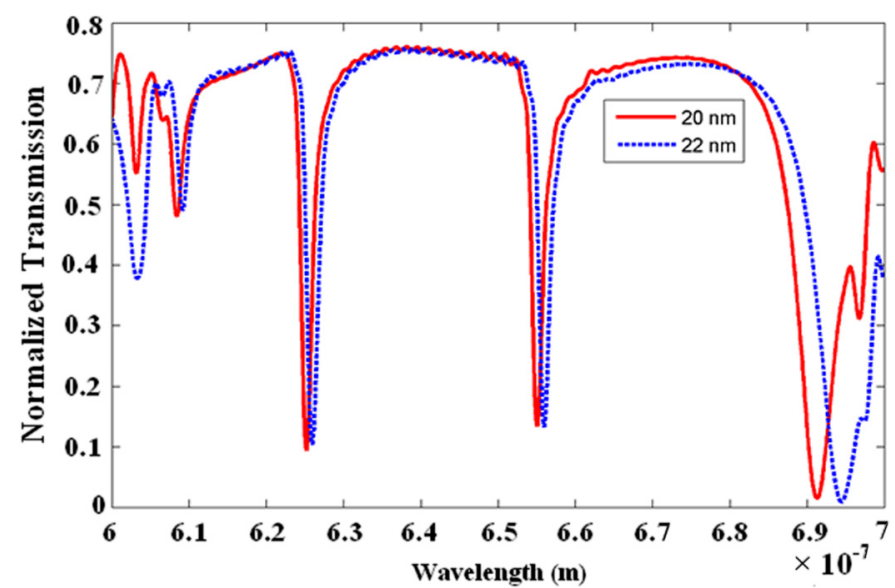

Fig. 7 Transmission intensity as a function of the wavelength for gap of $20 \mathrm{~nm}$ and gap of $22 \mathrm{~nm}$.

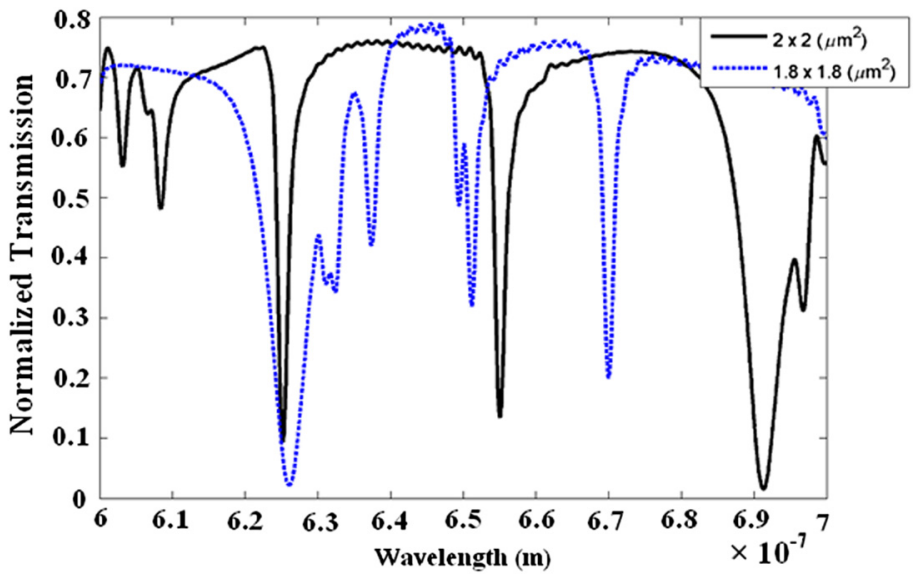

Fig. 8 Transmission intensity as a function of the wavelength for different square resonator sizes.

\section{Discussion and Conclusion}

We have proposed a sensing system using the plasmonic resonator for detecting microchanges of the refractive index. Our proposed sensor is compact and can easily be utilized for integrated on chip system for lab-on-chip application. The sensor is associated with a sensitivity of $700 \mathrm{~nm} / \mathrm{RIU}$ and a high detection limit, which could reach to $10^{-6}$ RIU. The sensor utilizes square shape resonator as the key sensing element. This resonator can be utilized as a size selector that detects particles with size smaller than its size only which can fit inside it. Thus, by 
controlling the size of this resonator, the upper limit of particle size that can be detected is also controlled. Our proposed sensor could be used in gas- and liquid-phase chemical sensing. In addition this sensor could be utilized for biosensing thus, by capturing of biomolecules producing a sensing signal that enables quantification of the biomolecules in a sample. The proposed structure opens the door for a high-sensitivity on-chip sensor using the plasmonic silicon hybrid technology toward practicable realization for lab-on-chip system.

\section{Acknowledgments}

The authors would like to acknowledge financial support received from the NEC C\&C Foundation of Japan and the support from the AUC.

\section{References}

1. W. Lukosz, "Principles and sensitivities of integrated optical and surface plasmon sensors for direct affinity sensing and immunosensing," Biosens. Bioelectron. 6(3), 215-225 (1991), http://dx.doi.org/10.1016/0956-5663(91)80006-J.

2. M. A. Cooper, "Optical biosensors in drug discovery," Nat. Rev. Drug Discov. 1(7), 515-528 (2002), http://dx.doi.org/10.1038/nrd838.

3. V. S.-Y. Lin et al., "A porous silicon-based optical interferometric biosensor," Science 278(5339), 840-843 (1997), http://dx.doi.org/10.1126/science.278.5339.840.

4. A. J. Haes and R. P. Van Duyne, "A nanoscale optical biosensor: sensitivity and selectivity of an approach based on the localized surface plasmon resonance spectroscopy of triangular silver nanoparticles," J. Am. Chem. Soc. 124(35), 10596-10604 (2002), http://dx.doi.org/ 10.1021/ja020393x.

5. J. Homola, "On the sensitivity of surface plasmon resonance sensors with spectral interrogation," Sens. Actuators B Chem. 41(1-3), 207-211 (1997), http://dx.doi.org/10.1016/ S0925-4005(97)80297-3.

6. J. Homola, S. S. Yee, and G. Gauglitz, "Surface plasmon resonance sensors: review," Sens. Actuators B 54(1-2), 3-15 (1999), http://dx.doi.org/10.1016/S0925-4005(98)00321-9.

7. M. E. Stewart et al., "Nanostructured plasmonic sensors," Chem. Rev. 108(2), 494-521 (2008), http://dx.doi.org/10.1021/cr068126n.

8. J. Homola, "Surface plasmon resonance based sensors," in Springer Series on Chemical Sensors and Biosensors, Vol. 4, Springer, Berlin (2006).

9. B. Min et al., "High-Q surface-plasmon-polariton whispering-gallery microcavity," Nature 457(7228), 455-458 (2009), http://dx.doi.org/10.1038/nature07627.

10. Q. Zhang et al., "A subwavelength coupler-type MIM optical filter," Opt. Express 17(9), 7549-7555 (2009), http://dx.doi.org/10.1364/OE.17.007549.

11. M. A. Swillam and A. S. Helmy, "Feedback effect in plasmonic slot waveguides examined using closed form model," Photonics Technol. Lett. 24(6), 497-499 (2012), http://dx.doi .org/10.1109/LPT.2011.2181350.

12. T.-B. Wang et al., "The transmission characteristics of surface plasmon polaritons in ring resonator," Opt. Express 17(26), 240961 (2009), http://dx.doi.org/10.1364/OE.17.024096.

13. S. Randhawa et al., "Experimental demonstration of dielectric-loaded plasmonic waveguide disk resonators at telecom wavelengths," Appl. Phys. Lett. 98(16), 161102 (2011), http:// dx.doi.org/10.1063/1.3574606.

14. Lumerical Solutions Inc., http://www.lumerical.com/tcad-products/fdtd/ (January 2013).

15. Z. Liu et al., "Near-field Moiré effect mediated by surface plasmon polariton excitation," Opt. Lett. 32(6), 629-631 (2007), http://dx.doi.org/10.1364/OL.32.000629.

16. P. Debackere et al., "Si based waveguide and surface plasmon sensors," Proc. SPIE 6477, 647719 (2007), http://dx.doi.org/10.1117/12.702040.

17. I. M. White and X. Fan, "On the performance quantification of resonant refractive index sensors," Opt. Express 16(2), 1021-1028 (2008), http://dx.doi.org/10.1364/OE.16.001020.

18. Z. Yu and S. Fan, "Extraordinarily high spectral sensitivity in refractive index sensors using multiple optical modes," Opt. Express 19(11), 10029-10040 (2011), http://dx.doi.org/ 10.1364/OE.19.010029. 
19. C. Lin et al., "Efficient broadband energy transfer via momentum matching at hybrid junctions of guided," Appl. Phys. Lett. 101(12), 123115 (2012), http://dx.doi.org/10.1063/ 1.4753985 .

20. B. Lau, M. A. Swillam, and A. S. Helmy, "Hybrid orthogonal junctions: wideband plasmonic slot-silicon wire couplers," Opt. Express 18(26), 27048-27059 (2010), http:// dx.doi.org/10.1364/OE.18.027048.

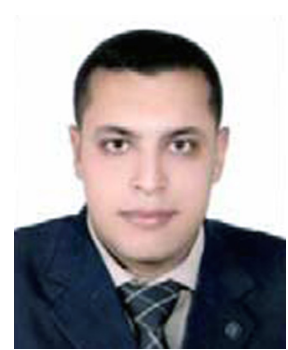

Salah E. El-Zohary received his bachelor's degree from Physics Department, Faculty of Science, Tanta University, Egypt in 2004, where he was appointed as demonstrator. In 2009, he joined the Department of Optical System Engineering, Graduate School of Advanced Technology and Science, The University of Tokushima, Japan, where he received his master's degree in 2011. From April 2011, he started as a doctoral student in the same laboratory in Japan. In 2012, he was appointed as assistant lecturer in Physics Department, Faculty of Science, Tanta University. His dominant research is focusing on novel nanofabrications for advanced nanophotonic and optoelectronic devices.

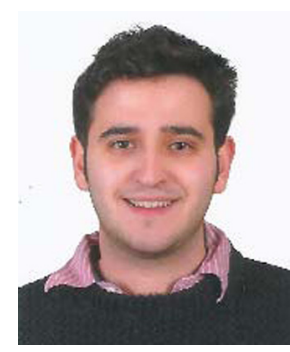

Abdulilah Azzazi graduated from Najd National Schools in 2009, and he is currently an undergraduate student at American University in Cairo, majoring in physics and mathematics, minoring in computer science. He is keen on learning about formal logic, computer architecture, and photonics and is avid about digital optical computing. He joined Dr. Swillam's research group in the winter of 2012 and has since participated in multiple projects.

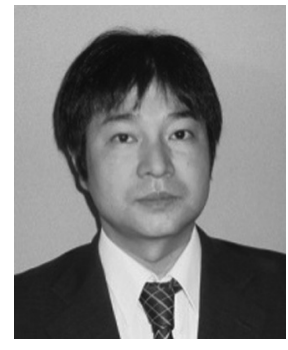

Hiroyuki Okamoto received his BE, ME, and Dr Eng. degrees in electronic engineering from the University of Tokushima in 1992, 1994, and 2003, respectively. He joined Anan National College of Technology as a research associate in 2004 and is now an associate professor. He is engaged in research on an optical device using the microring resonator.

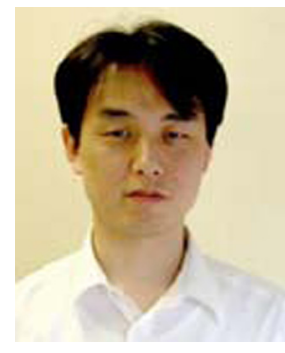

Toshihiro Okamoto received his BE and ME degrees in electronic engineering from the University of Tokushima in 1991 and 1993 and his Dr. Eng. degree from the University of Tokushima in 2000 . He is an assistant professor at the University of Tokushima from 1994. His dominant research areas are nonlinear phenomena and its application of surface plasmons.

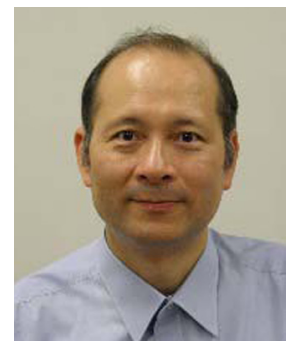

Masanobu Haraguchi received his $\mathrm{PhD}$ degree from Osaka University in 1993. From 2009, he is a professor at the University of Tokushima. He is interested in characteristics and applications of localized and propagating plasmons at nanometal structure. Recently, his main interests are channeltype plasmonic waveguides, their applications, and a split ring resonator for NIR and visible region. 


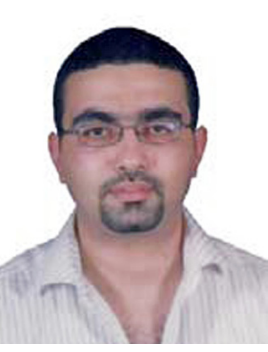

Mohamed Swillam received his $\mathrm{PhD}$ from McMaster University, Hamilton, Canada, in 2008. After graduation, he worked as a postdoctoral fellow in the same group. In October 2009, he joined the photonic group and the Institute of Optical Sciences at the University of Toronto, where he works as a research fellow. In September 2011, he was appointed as an assistant professor at the Department of Physics, the American University in Cairo (AUC). His research interests include active and passive nanophotonic and plasmonic devices and systems, silicon photonics, metamaterials, and solar cells. He authored more than 80 technical papers in highly ranked journals and conferences in these areas. 